\title{
How We Use Reticulocyte Parameters in Workup and Management of Pediatric Hematologic Diseases
}

\author{
Emilia Parodi ${ }^{1 \dagger}$, Francesca Romano ${ }^{2 * \dagger}$ and Ugo Ramenghi ${ }^{1,2}$ \\ ${ }^{1}$ Pediatric Hematology, Department of Pediatric and Public Health Sciences, University of Torino, Turin, Italy, ${ }^{2}$ Postgraduate \\ School of Pediatrics, University of Torino, Turin, Italy
}

Keywords: reticulocyte, $\mathrm{CHr}$, ARC, iron deficiency anemia, anemia, pediatric, newborn

\section{INTRODUCTION}

OPEN ACCESS

Edited by:

Seth Corey,

Cleveland Clinic, United States

Reviewed by:

Gary Kupfer,

Georgetown University Medical

Center, United States

Ravi Talati,

Cleveland Clinic Children's,

United States

*Correspondence:

Francesca Romano

francesca_romano@hotmail.com

†These authors have contributed equally to this work

Specialty section: This article was submitted to

Pediatric Hematology and Hematological Malignancies,

a section of the journal

Frontiers in Pediatrics

Received: 29 July 2020 Accepted: 13 November 2020 Published: 04 December 2020

Citation:

Parodi E, Romano F and Ramenghi U (2020) How We Use Reticulocyte

Parameters in Workup and Management of Pediatric Hematologic

Diseases. Front. Pediatr. 8:588617. doi: 10.3389/fped.2020.588617
Reticulocyte parameters are simple to obtain, require a very small extra amount of blood and provide additional information about erythrocyte production. However, their use in pediatric routine care is still limited.

Aim of the present opinion paper is to describe the usefulness of these tools for Pediatricians in the workup and management of pediatric hematologic diseases.

\section{RETICULOCYTE PARAMETERS}

\section{Reticulocyte Physiology}

Reticulocytes are the youngest erythrocytes that the bone marrow releases into peripheral blood. Thus, they represent a reliable index of recent bone marrow erythrocyte production.

Under normal conditions, nucleated erythroid precursor cells clonally mature in 1-3 days within the bone marrow. When their nucleus is extruded from the cell, the young erythrocytes (i.e., reticulocytes) are released into peripheral blood where they circulate for 1-2 days, before becoming mature erythrocytes. Reticulocytes can be identified through specific staining (manual reticulocyte count) or through quantification of their residual RNA (automated reticulocyte count) (1).

\section{Absolute Reticulocyte Count (ARC)}

Usually, the reticulocyte reference range is reported as a percentage of the red blood cells total. After the first few months of life, the normal reticulocyte percentage in children is equal to the adult's one $(\sim 1.5$ percent $)(2)$.

However, in patients with anemia, the reticulocyte percentage must be interpreted in relation to the reduced number of red blood cells. For example, in children affected by aplastic anemia, a normal reticulocyte percentage does not reflect a proper bone marrow production.

The absolute reticulocyte count (ARC), defined as the number of reticulocytes $/ \mu \mathrm{L}$, better reflects bone marrow function and effective erythropoiesis. A normal ARC both in pediatric (after the first 3 months of life) and adult age is $\sim 25,000$ to $75,000 / \mu \mathrm{L}(1.0 \pm 0.5$ percent of the 5 million red cells $/ \mu \mathrm{L}$ ) and is now calculated and reported by many automated cell counters (2).

\section{New Reticulocyte Parameters: The Reticulocyte Hemoglobin Content (CHr)}

Modern analyzers and flow cytometric techniques, although not readily available in all institutions, can measure red blood cell and reticulocyte features more accurately than in the past, without relevant additional costs. 
The reticulocyte hemoglobin content $(\mathrm{CHr})$ is the product of the cellular volume and the cellular hemoglobin concentration. $\mathrm{CHr}$ is obtained on a very small sample of blood as part of the complete blood count and does not require additional phlebotomy (3). Since erythrocytes have a slow turnover (120 days), their indices are not efficient indicators of early iron deficient erythropoiesis. On the contrary, as reticulocytes circulate for only 1-2 days, $\mathrm{CHr}$ provides a reliable measure of the functional iron available for new red blood cell production over the previous 3-4 days and proved to be sensitive indicator of early iron deficient erythropoiesis.

\section{Reticulocyte Parameters in the Newborn}

After birth and until 8-12 weeks, hemoglobin physiologically falls due to the suppression of erythropoietin in the extrauterine hyperoxic environment. This drop is more dramatic in preterm infants. At birth, the reticulocyte count is higher than at any time during healthy life; ARC abruptly falls during the first days after birth, following a fall in erythropoietin production. A slight upturning in reticulocyte count is expected in the subsequent 3 weeks; after the first 3 months of life ARC reaches the normal reference range for pediatric and adult population (4).

Even though there are no standardized cut offs in the newborn, $\mathrm{CHr}$ is a good marker of iron status also in the first days and months of life, even in preterm and critically ill babies.

\section{DISCUSSION}

We will review and discuss the usefulness of reticulocyte parameters in the diagnosis and management of pediatric hematologic diseases.

\section{Absolute Reticulocyte Count (ARC) in the Diagnosis of Pediatric Anemia}

As the reticulocyte count distinguishes disorders resulting from rapid destruction or loss of erythrocytes from disorders resulting from bone marrow failure, we strongly suggest its use in the initial diagnostic workout of pediatric anemia.

$\checkmark$ High reticulocyte count: consider hemolysis (i.e., red blood cells membrane defects, enzymatic defects, autoimmune diseases, sickle cell disease), or bleeding

$\checkmark$ Low reticulocyte count: consider bone marrow depression (i.e., infections, transient erythroblastopenia of childhood, congenital bone marrow failures)

$\checkmark$ Note: Parvovirus B19 can cause transient aplastic crisis in patients with chronic hemolytic diseases (i.e., spherocytosis, sickle cell disease, thalassemia trait, congenital dyserythropoietic anemia) resulting in severe acute anemia. In these patients, ARC is a very useful index in identifying transient marrow suppression and the need of transfusion.

Abbreviations: ARC, Absolute reticulocyte count; $\mathrm{CHr}$, Reticulocyte Hemoglobin Content; Hb, Hemoglobin; MCV, Mean Cellular Volume; NICU, Neonatal Intensive Care Unit.

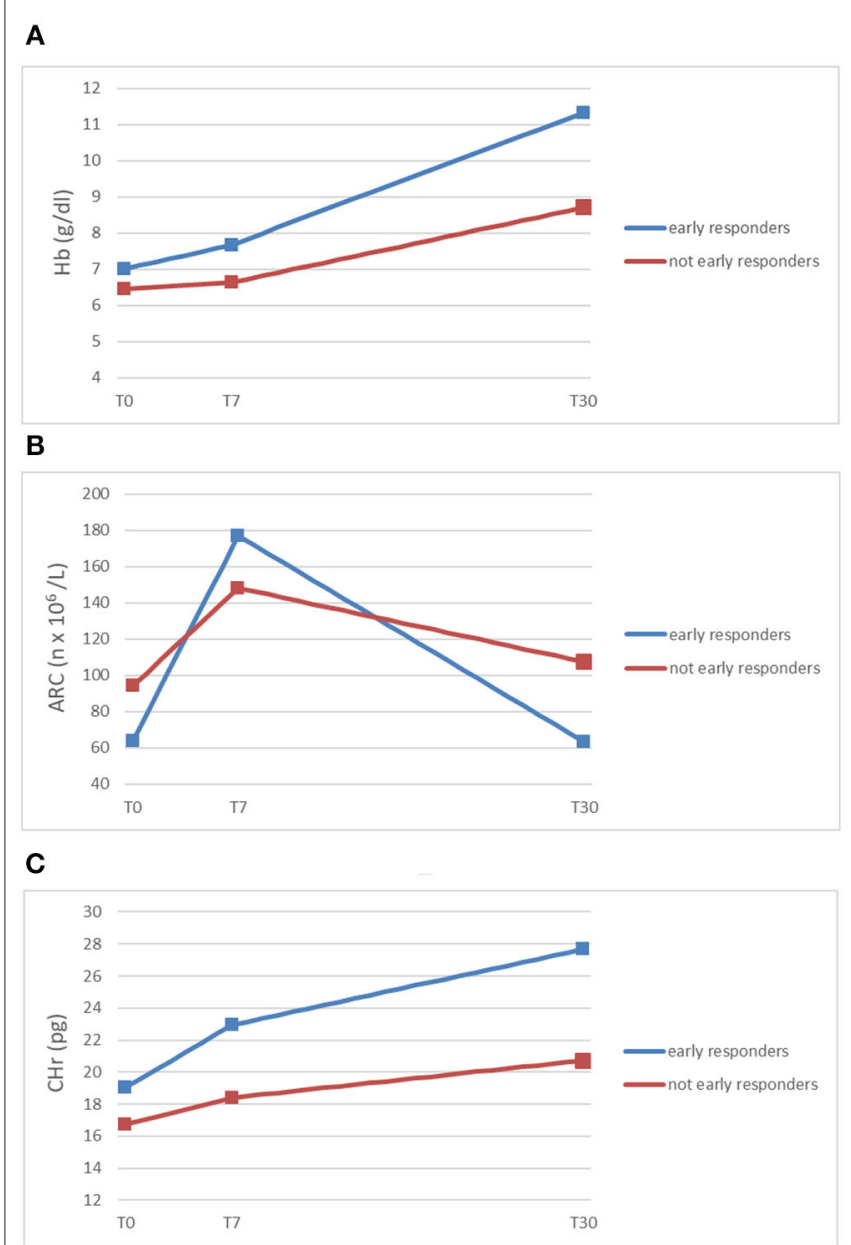

FIGURE 1 | Trends of mean $\mathrm{Hb}$ (A), ARC (B), and $\mathrm{CHr}$ (C) (y-axis) in early responding patients and not early responding patients at diagnosis (TO), within 7 days from the beginning of oral iron supplementation (T7), and after 30 days (T30) $(x$-axis) $(5,6)$.

\section{Absolute Reticulocyte Count (ARC) in the Follow up of Pediatric Anemia}

ARC reflects both spontaneous bone marrow recovery (for e.g., after transient bone marrow suppression caused by a viral infection) and response to therapy in iron deficiency anemia.

We previously identified ARC as an accurate marker to early detect response to exclusive oral iron supplementation (iron sulfate $2 \mathrm{mg} / \mathrm{kg} / \mathrm{die}$ ) in a cohort of children with severe iron deficiency anemia. A significant association was demonstrated between relative increase of $\mathrm{ARC}$ at day +3 and $\mathrm{Hb}$ at day +14 (5).

Subsequently, we demonstrated the relative increase of ARC at day +7 to be predictive of complete early response (normal hemoglobin value per age and sex at day +30) (Figure 1) (6).

\section{Reticulocyte Hemoglobin Content (CHr) in the Diagnosis of Iron Deficiency Anemia}

$\mathrm{CHr}$ has been validated in literature as the strongest independent predictor of iron deficiency and iron deficiency anemia, 
when compared to other parameters (hemoglobin, ferritin, transferrin saturation, or mean corpuscular volume-MCV), both in adults and children. In pediatric age, optimal $\mathrm{CHr}$ cutoff was established at $27.5 \mathrm{pg}$ for detecting iron deficiency (sensitivity $83 \%$ and specificity $72 \%$ ) (7) and $26 \mathrm{pg}$ for detecting iron deficiency anemia (sensitivity $83 \%$ and specificity 75\%) (8).

However, since $\mathrm{MCV}$ is used to calculate $\mathrm{CHr}$, this index has diagnostic limitations in patients with other causes of altered mean MCV (it is low in iron-replete patients with hemoglobinopathies associated with microcytosis and it can be normal or elevated in iron-deficient patients with high MCV, for example in patients with both iron and Vitamin B12 deficiency) (9).

\section{Reticulocyte Hemoglobin Content (CHr) in the Follow Up of Iron Deficiency Anemia}

$\mathrm{CHr}$ has been shown to be an accurate marker in monitoring response to iron therapy in adults and in pediatric patients receiving hemodialysis (3).

For the first time in literature, we demonstrated $\mathrm{CHr}$ to be the earliest marker of response to oral iron treatment $(48 \mathrm{~h})$ in children with severe iron deficiency anemia (5).

Moreover, $\mathrm{CHr}$ measured within 7 days from the start of oral iron resulted significantly higher in children who reached reference value of hemoglobin $(\mathrm{Hb})$ concentration for age and sex at day +30 from the beginning of treatment (6).

As shown in Figure 1, ARC and CHr are early and accurate predictors of response to oral therapy in iron deficiency anemia. Early detection of patients not responding to oral iron (malabsorption? persistent blood loss?) is crucial to switch them to different diagnostic workout and/or treatment, such as parenteral iron supplementation or transfusion.

\section{Reticulocyte Parameters and the Newborn}

Both anemia of prematurity and iron deficiency anemia in early stages of life can affect long term infants' growth and neurologic development. Thus, it is essential for the pediatrician to promptly detect and treat these conditions and to take advantage of easy tools like reticulocyte parameters also in the first days and months of life.

Iron stores in the newborn mainly depend on maternal stores, gestational age (most of the transfer of maternal iron to the fetus occurs during the third trimester of gestation) timing of cordon clamping [delayed cordon clamping, i.e., not earlier than $1 \mathrm{~min}$ after birth, improves iron status in the child (10)] and timing of introduction of complementary food. In term healthy children, iron is usually sufficient up to when the child has doubled its weight (2), which usually occurs between the 4 th and the 6 th month of life. The introduction of complementary food after 6 months of age exposes the child to iron deficiency especially in exclusive breastfed infants $(11,12)$. Particularly, late preterm babies represent an underestimated category at risk of iron deficient anemia (13).

$\mathrm{CHr}$ could be a simple and reliable index to detect iron deficiency both in the newborn and in late preterm infants and low birth weight infants on exclusive breastfeeding, especially in those with an important catchup growth and delay in introduction of complementary food. Compared to other indices, it requires less blood and it is not affected by gestational age, stress, and inflammation.

Some authors suggested $25 \mathrm{pg}$ as cut off for iron deficiency within the first $24 \mathrm{~h}$ of life (14), others $30 \mathrm{pg}$ (15). In the first months of life, some authors set the cut off at $27 \mathrm{pg}$, for both term and preterm infants $(16,17)$.

We recently conducted a survey on iron prophylaxis in Neonatal Units and NICUs of three Italian regions, underlining an important variability between the Centers in prescription of iron prophylaxis (dose, duration, type of iron). A few NICUs currently use $\mathrm{CHr}$ to modulate initial dose of iron supplementation in preterm babies and less than a quarter of late preterm babies received iron prophylaxis, affirming the need for practical guidelines for Neonatologists (18).

\section{CONCLUSIONS}

Reticulocyte parameters are obtained on a very small sample of blood as part of the complete blood count and do not require additional phlebotomy.

ARC can distinguish anemia resulting from rapid destruction or loss of red blood cells from disorders causing bone marrow depression.

$\mathrm{ARC}$ and $\mathrm{CHr}$ are useful in the diagnosis of iron deficiency and iron deficiency anemia, in both children and newborn, and they are reliable and early predictor of response to oral iron therapy.

Reticulocyte parameters provide useful information to Pediatricians and Neonatologists for the diagnosis and the management of hematologic diseases. Don't forget to take advantage of them!

\section{AUTHOR CONTRIBUTIONS}

EP and FR gave substantial contribution to conception, drafted the article, reviewed and revised the manuscript. UR critically reviewed and revised the manuscript. All authors approve the final version of the manuscript as submitted and agree to be accountable for all aspects of the work.

\section{FUNDING}

This work was partially supported by Banca del Piemonte grant to UR. 


\section{REFERENCES}

1. Piva E, Brugnara C, Spolaore F, Plebani M. Clinical utility of reticulocyte parameters. Clin Lab Med. (2015) 35:133-63. doi: 10.1016/j.cll.2014.10.004

2. Arceci R, Hann IM, Smith OP. Pediatric Hematology. Blackwell (2006). 826 p.

3. Mast AE, Blinder MA, Dietzen DJ. Reticulocyte hemoglobin content. Am J Hematol. (2008) 83:307-10. doi: 10.1002/ajh.21090

4. Christensen RD, Henry E, Jopling J, Wiedmeier SE. The CBC: reference ranges for neonates. Semin Perinatol. (2009) 33:3-11. doi: 10.1053/j.semperi.2008.10.010

5. Parodi E, Giraudo MT, Davitto M, Ansaldi G, Mondino A, Garbarini L, et al. Reticulocyte parameters: markers of early response to oral treatment in children with severe iron-deficiency anemia. J Pediatr Hematol Oncol. (2012) 34:e249-52. doi: 10.1097/MPH.0b013e3182588996

6. Parodi E, Giraudo MT, Ricceri F, Aurucci ML, Mazzone R, Ramenghi U. Absolute reticulocyte count and reticulocyte hemoglobin content as predictors of early response to exclusive oral iron in children with iron deficiency anemia. Anemia. (2016) 2016:7345835. doi: 10.1155/2016/7345835

7. Brugnara C, Zurakowski D, DiCanzio J, Boyd T, Platt O. Reticulocyte hemoglobin content to diagnose iron deficiency in children. JAMA. (1999) 281:2225-30. doi: 10.1001/jama.281.23.2225

8. Ullrich C, Wu A, Armsby C, Rieber S, Wingerter S, Brugnara C, et al. Screening healthy infants for iron deficiency using reticulocyte hemoglobin content. JAMA. (2005) 294:924-30. doi: 10.1001/jama.294.8.924

9. Balci YI, Akpinar FO, Polat A, Uzun U, Ergin A. Evaluation of reticulocyte parameters in iron deficiency, vitamin B12 deficiency and mixed anemia. Clin Lab. (2016) 62:343-7. doi: 10.7754/clin.lab.2015.150616

10. Committee on Obstetric Practice, American College of Obstetricians and Gynecologists. Committee opinion no.543: timing of umbilical cord clamping after birth. Obstet Gynecol. (2012) 120:1522-6. doi: 10.1097/01.AOG.0000423817.471 65.48

11. Baker RD, Greer FR. Diagnosis and prevention of iron deficiency and irondeficiency anemia in infants and young children ( $0-3$ years of age). Pediatrics. (2010) 126:1040-50. doi: 10.1542/peds.2010-2576

12. Agostoni C, Buonocore G, Carnielli VP, De Curtis M, Darmaun D, Decsi T, et al. Enteral nutrient supply for preterm infants: commentary from the European society of paediatric gastroenterology, hepatology and nutrition committee on nutrition. J Pediatr Gastroenterol Nutr. (2010) 50:8591. doi: 10.1097/MPG.0b013e3181adaee0

13. Yamada RT, Leone CR. Hematological and iron content evolution in exclusively breastfed late-preterm newborns. Clinics. (2014) 69:7928. doi: 10.6061/clinics/2014(12)01

14. Lorenz L, Arand J, Buchner K, Wacker-Gussmann A, Peter A, Poets CF, et al. Reticulocyte haemoglobin content as a marker of iron deficiency. Arch Dis Child Fetal Neonatal Ed. (2015) 100:F198-202. doi: 10.1136/archdischild-2014-306076

15. Christensen RD, Henry E, Bennett ST, Yaish HM. Reference intervals for reticulocyte parameters of infants during their first 90 days after birth. $J$ Perinatol. (2016) 36:61-6. doi: 10.1038/jp.2015.140

16. Al-Ghananim RT, Nalbant D, Schmidt RL, Cress GA, Zimmerman MB, Widness JA. Reticulocyte hemoglobin content during the first month of life in critically ill very low birth weight neonates differs from term infants, children, and adults. J Clin Lab Anal. (2016) 30:326-34. doi: 10.1002/jcla.21859

17. Morton SU, Yuen JC, Feldman HA, Hashim E, Rudie C, Lindamood $\mathrm{KE}$, et al. Screening with reticulocyte hemoglobin increased iron sufficiency among NICU patients. Pediatr Qual Saf. (2020) 5:e258. doi: 10.1097/pq9.0000000000000258

18. Parodi E, Ferrero A, Perrone B, Saracco P, Teresa M, Regoli D. ScienceDirect current practice of iron prophylaxis in preterm and low birth weigh neonates: a survey among Italian neonatal units. Pediatr Neonatol. (2018) 59:581-5. doi: 10.1016/j.pedneo.2018.01.013

Conflict of Interest: The authors declare that the research was conducted in the absence of any commercial or financial relationships that could be construed as a potential conflict of interest.

Copyright (๑) 2020 Parodi, Romano and Ramenghi. This is an open-access article distributed under the terms of the Creative Commons Attribution License (CC BY). The use, distribution or reproduction in other forums is permitted, provided the original author(s) and the copyright owner(s) are credited and that the original publication in this journal is cited, in accordance with accepted academic practice. No use, distribution or reproduction is permitted which does not comply with these terms. 